\title{
State Estimation and Parameter Identification of Flexible Manipulators Based on Visual Sensor and Virtual Joint Model
}

\author{
Department of Mechanical Engineering \\ Kyoto University \\ Kyoto 606-8501, Japan
}

Tsuneo YOSHIKAWA, Atsuharu OHTA ${ }^{\dagger}$ and Katsuya KANAOKA ${ }^{\ddagger}$

\begin{abstract}
When we control a flexible manipulator based on a dynamic model, we need to estimate the state variables with accuracy. In the case of rigid manipulators, the state variables consist of joint angles and their velocities, which are measured easily by encoders, potentiometers, tachometers, and so on. In the case of flexible manipulators, however, the state variables also include elastic deformations and their velocities due to flexibility.

Here we focus on visual sensor to measure distributed state variables of flexible manipulators. First, we discuss a distributed state estimation method which uses discrete position information of markers attached on flexible links. Second, when we apply the virtual joint model to flexible manipulators, elastic deformations are expressed by virtual passive joint angles which are lumped. So we have to correspond the real elastic deformations to the virtual joint angles. We also discuss this transformation. Third, when we estimate the lumped state variables by the proposed method, it is possible to identify physical parameters of the dynamic model. Finally, some experiments were carried out to verify the effectiveness of the proposed method.
\end{abstract}

\section{Introduction}

Generally, for precise control or analysis of dynamic motion of a flexible manipulator, it is essential to have a dynamic model of flexibility $[1,2,3]$. As one of the modeling methods of flexible manipulators, the virtual joint model were proposed by Yoshikawa et al. [4]. Parameter identification and manipulator control based on the virtual joint model have been studied $[4,5]$.

\footnotetext{
*yoshi@mech.kyoto-u.ac.jp

†atsuharu@ota.tec.toyota.co.jp, currently at TOYOTA Motor Corporation, 1200 Mishuku, Susono-shi, Shizuoka 410-1107, Japan

${ }^{\ddagger} \mathrm{t} 60 \mathrm{v} 1546 @$ ip.media.kyoto-u.ac.jp
}

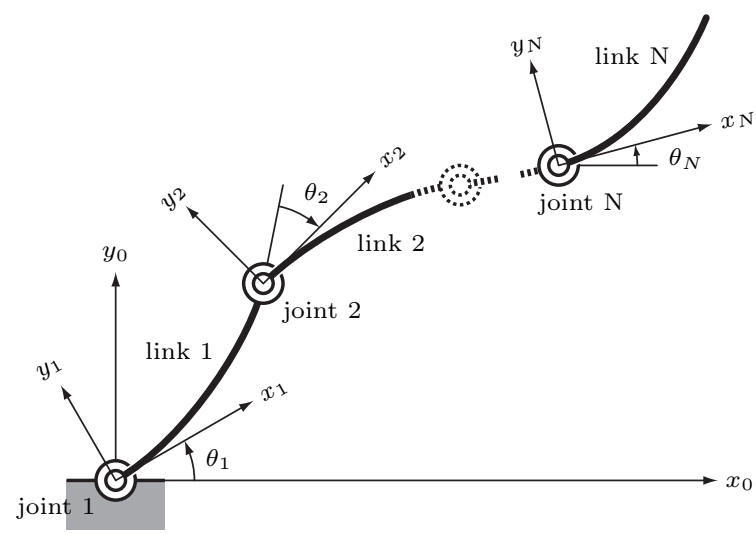

Figure 1: Flexible manipulator

In a previous research [5], the state observer was used for state estimation of this dynamic model. However, precise state estimation by this method becomes difficult when the virtual link division number is large, which is the case that the links have much more flexibility.

In this paper, we propose a state estimation method based on discrete position information of flexible links by visual sensor. Some previous research [6] used visual sensors to control flexible manipulators, but the visual sensors were utilized just to measure the tip position of flexible link, not to estimate the state variables directly.

\section{Flexible Manipulator}

We consider a flexible manipulator shown in Fig.1 in this paper. It is $N$ d.o.f. plane manipulator which has serial $N$ flexible links and $N$ joints, and fixed on the inertial coordinate system $\Sigma_{0}$.

Each joint displacement is $\boldsymbol{\theta}=\left[\theta_{1}, \theta_{2}, \cdots, \theta_{N}\right]^{T}$. The length of link $i(i=1,2, \cdots, N)$ without elastic deformation is $l_{i}$, and the mass is $m_{i}$. 


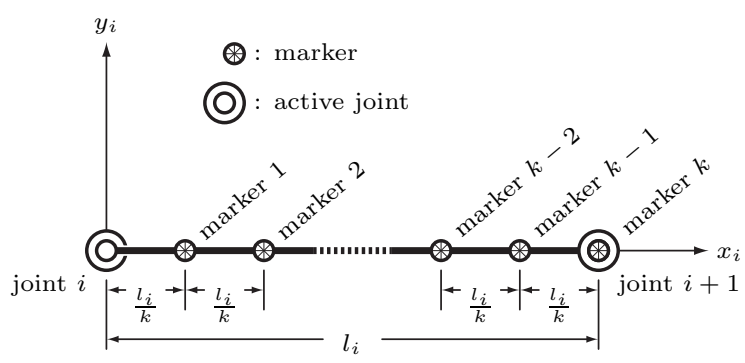

Figure 2: Markers on the flexible link $i$

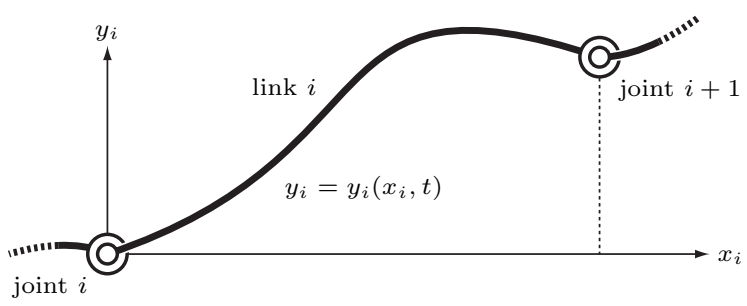

Figure 3: Flexible link shape function

The link $i$ coordinate system $\Sigma_{i}$ is fixed on the center of joint $i$. Its $x_{i}$ axis is along link $i$ when it's not deformed, $z_{i}$ axis is along the displacement axis of joint $i$, and $y_{i}$ axis is determined to compose the right hand coordinate system.

\section{Distributed State Estimation Based on Visual Sensor}

In this section, we describe a method utilizing a visual sensor to estimate distributed state variables of flexible manipulators.

In this method, only visual markers are attached on flexible links, so the system of manipulator side becomes simple compared to other methods, such as strain gages or accelerometers. The visual sensor measures position information, which is not differential value, we don't have to consider its integration error. If more precision is needed, we can increase the points of measurement easily. If two or more cameras are available, we can get 3-dimensional information of markers to extend this method.

\subsection{Attaching Markers}

In this method, $k$ markers are attached on link $i$ as shown in Fig.2. As we mention in following Section 3.2 and 3.3 , it is essential the number of markers enough to determine a flexible link shape function.

If we know the minimum wave length $\lambda_{\text {min }}$ which composes the link shape by physical consideration such as natural frequencies, the minimum $k$ is constrained by the sampling theorem.

$$
k \geq \frac{2 l_{i}}{\lambda_{\min }}
$$

\subsection{Flexible Link Shape Function}

The elastic deformation $y_{i}\left(x_{i}, t\right)$ of link $i$ and its velocity $v_{i}\left(x_{i}, t\right)$ at time $t$ are distributed state variables (see Fig.3).

We choose a set of approximation functions to estimate this state variables from discrete position information of markers. We call this set the flexible link shape function. Here we adopt polynomial approximation as the link shape function :

$$
y_{i}\left(x_{i}, t\right)=\sum_{j=1}^{o_{\max }} a_{i j}(t) x_{i}{ }^{j}
$$

where $o_{\max }$ is the maximum order of the polynomial.

This $o_{\max }$ may cause improper approximation if it is too large. In many cases $o_{\max }$ must be smaller than 4 or 5 , though it depends on the marker number $k$. If the link is so flexible that $o_{\max }$ order polynomial does not reflect the real link shape, we should adopt another one as the link shape function, for instance, trigonometric function.

\subsection{Fitting Link Shape Function}

Consider the polynomial function (2). When the position information of $k$ markers on link $i$

$$
{ }^{i} \boldsymbol{p}_{j}(t)=\left[\begin{array}{c}
{ }^{i} p_{x j}(t) \\
{ }^{i} p_{y j}(t)
\end{array}\right] \quad(j=1,2, \cdots, k)
$$

is measured by a visual sensor, the measured values matrix ${ }^{i} \boldsymbol{P}(t)$, the measured values vector ${ }^{i} \boldsymbol{Y}(t)$, and coefficients vector of the link shape function $\boldsymbol{a}_{i}(t)$ are defined as follows.

$$
\begin{aligned}
{ }^{i} \boldsymbol{P}(t) & =\left[\begin{array}{cccc}
{ }^{i} p_{x 1} & { }^{i} p_{x 1}{ }^{2} & \ldots & { }^{i} p_{x 1}{ }^{\text {omax }} \\
{ }^{i} p_{x 2} & { }^{i} p_{x 2}{ }^{2} & \ldots & { }^{i} p_{x 2}^{\text {omax }} \\
\vdots & \vdots & \ddots & \vdots \\
{ }^{i} p_{x k} & { }^{i} p_{x k}{ }^{2} & \ldots & { }^{i} p_{x k}{ }^{\text {omax }}
\end{array}\right] \\
{ }^{i} \boldsymbol{Y}(t) & =\left[\begin{array}{llll}
{ }^{i} p_{y 1} & { }^{i} p_{y 2} & \ldots & { }^{i} p_{y k}
\end{array}\right]^{T} \\
\boldsymbol{a}_{i}(t) & =\left[\begin{array}{llll}
a_{i 1} & a_{i 2} & \cdots & a_{\text {iomax }}
\end{array}\right]^{T}
\end{aligned}
$$

Then the coefficients vector $\boldsymbol{a}_{i}(t)$ are estimated by the least square method.

$$
\boldsymbol{a}_{i}(t)=\left\{\left({ }^{i} \boldsymbol{P}\right)^{T}\left({ }^{i} \boldsymbol{P}\right)\right\}^{-1}\left({ }^{i} \boldsymbol{P}\right)^{T}\left({ }^{i} \boldsymbol{Y}\right)
$$




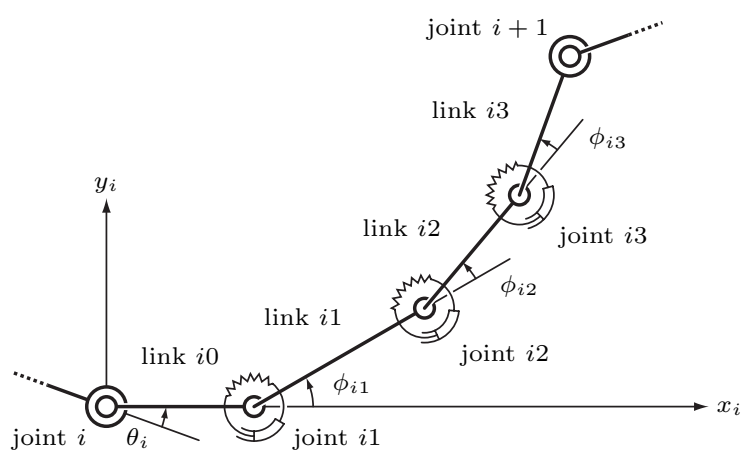

Figure 4: Construction of virtual joint model $(n=3)$

\section{Virtual Joint Model}

In this section, we describe a model construction of flexible manipulators based on the virtual joint model by Yoshikawa et al. [4]

\subsection{Model Construction}

In the virtual joint model, which is one of the lumped parameter models, each flexible link is divided into some virtual rigid links connected by virtual passive joints. The link flexibility is represented by virtual springs and dampers attached to the virtual passive joints.

As shown in Fig.4, each of $N$ flexible links is divided by $n$ virtual passive joints. The divided virtual rigid links are numbered link $i 0, i 1, \cdots$, in from the manipulator base (Fig.4 is an example of $n=3) . l_{i j}$ is the length, $l_{g i j}$ is the center of mass, $m_{i j}$ is the mass, $I_{i j}$ is the inertia moment around the center of mass of link ij $(j=0,1, \cdots, n)$.

The virtual passive joint attached to the base of link $i j(j=1,2, \cdots, n)$ is called joint $i j$. The displacement of the virtual passive joints on link $i$ is $\phi_{i}(t)=\left[\phi_{i 1}, \phi_{i 2}, \cdots, \phi_{i n}\right]^{T}$. The damping coefficient and spring constant of joint $i j$ are respectively $D_{i j}$, $K_{i j}$.

\subsection{Dynamics}

The equations of motion of a flexible manipulator based on the virtual joint model are given by

$$
\begin{aligned}
& \boldsymbol{M}_{11}(\boldsymbol{\psi}) \ddot{\boldsymbol{\theta}}+\boldsymbol{M}_{12}(\boldsymbol{\psi}) \ddot{\boldsymbol{\phi}}+\boldsymbol{h}_{1}(\boldsymbol{\psi}, \dot{\boldsymbol{\psi}})=\boldsymbol{\tau} \\
& \boldsymbol{M}_{21}(\boldsymbol{\psi}) \ddot{\boldsymbol{\theta}}+\boldsymbol{M}_{22}(\boldsymbol{\psi}) \ddot{\boldsymbol{\phi}}+\boldsymbol{h}_{2}(\boldsymbol{\psi}, \dot{\boldsymbol{\psi}})+\boldsymbol{D} \dot{\boldsymbol{\phi}}+\boldsymbol{K} \boldsymbol{\phi}=\mathbf{0}
\end{aligned}
$$

$\boldsymbol{M}_{11}, \quad \boldsymbol{M}_{12}, \quad \boldsymbol{M}_{21}, \boldsymbol{M}_{22}$ are the inertia matrices, $\boldsymbol{h}_{1}, \boldsymbol{h}_{2}$ are the vectors containing centrifugal, Coriolis, gravity and other nonlinear force, $\quad \boldsymbol{D}=\operatorname{diag}\left[D_{11}, D_{12}, \cdots, D_{N n}\right], \quad \boldsymbol{K}=$

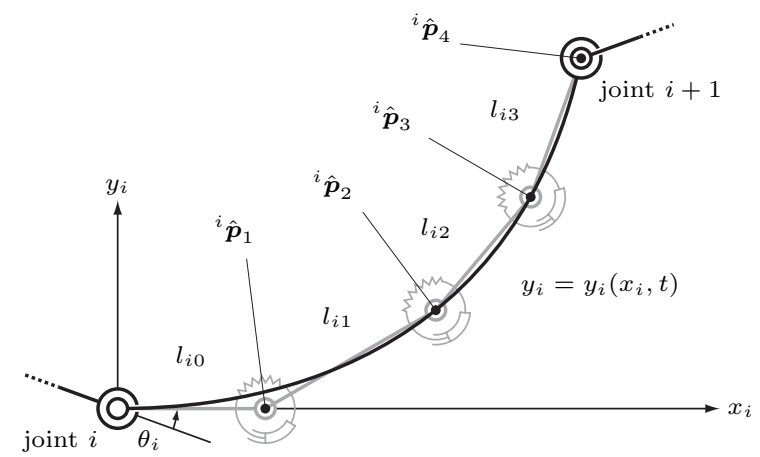

Figure 5: Fitting into virtual joint model $(\mathrm{n}=3)$

$\operatorname{diag}\left[K_{11}, K_{12}, \cdots, K_{N n}\right]$ are respectively the diagonal matrices of damping coefficients and spring constants. $\tau$ is the joint driving force. $\phi=\left[\phi_{1}{ }^{T}, \phi_{2}{ }^{T}, \cdots, \phi_{N}{ }^{T}\right]^{T}$ is the vector of passive joint angles, $\boldsymbol{\psi}=\left[\boldsymbol{\theta}^{T}, \boldsymbol{\phi}^{T}\right]^{T}$ is the vector of all joint angles.

\section{Lumped State Estimation of Virtual Joint Model}

In this section, we describe a method to transform the distributed state variables of flexible links into lumped state variables of the virtual joint model. When we get the lumped state variables by the proposed method, it is also possible to identify physical parameters of the dynamic model.

\subsection{Transformation into Lumped State Variables}

Here we set the position of virtual passive joints and link tip as the representative points of link $i$. ${ }^{i} \hat{\boldsymbol{p}}_{1}(t), \cdots,{ }^{i} \hat{\boldsymbol{p}}_{n+1}(t)$ denote the position of representative points of link $i$.

As shown in Fig.5, the representative point ${ }^{i} \hat{\boldsymbol{p}}_{j}(t)=\left[{ }^{i} \hat{p}_{x j},{ }^{i} \hat{p}_{y j}\right]^{T}(j=2, \cdots, n+1)$ is determined to locate on the link $i$ shape function $y_{i}\left(x_{i}, t\right)$. First, ${ }^{i} \hat{\boldsymbol{p}}_{1}(t)$ is given by

$$
{ }^{i} \hat{\boldsymbol{p}}_{1}(t)=\left[\begin{array}{c}
l_{i 0} \\
0
\end{array}\right]
$$

then, ${ }^{i} \hat{\boldsymbol{p}}_{j}(t)(j=2, \cdots, n+1)$ is determined as the point satisfying the following simultaneous equations.

$$
\begin{aligned}
& { }^{i} \hat{p}_{y j}=y_{i}\left({ }^{i} \hat{p}_{x j}, t\right) \\
& \left({ }^{i} \hat{p}_{x j}-{ }^{i} \hat{p}_{x, j-1}\right)^{2}+\left({ }^{i} \hat{p}_{y j}-{ }^{i} \hat{p}_{y, j-1}\right)^{2}=l_{i, j-1}{ }^{2}
\end{aligned}
$$

From these representative points, the virtual pas- 
sive joint angles $\phi_{i}(t)$ are given by

$$
\phi_{i j}(t)=\tan ^{-1}\left(\frac{{ }^{i} \hat{p}_{y, j+1}-{ }^{i} \hat{p}_{y j}}{{ }^{i} \hat{p}_{x, j+1}-{ }^{i} \hat{p}_{x j}}\right)-\sum_{j^{\prime}=1}^{j-1} \phi_{i j^{\prime}}
$$

The virtual passive joint velocity $\dot{\phi}_{i}(t)=$ $\left[\dot{\phi}_{i 1}, \dot{\phi}_{i 2}, \cdots, \dot{\phi}_{i n}\right]^{T}$ is estimated by numerical differentiation of $\phi_{i}(t)$ with respect to time $t$.

The transformation matrix ${ }^{i+1} \boldsymbol{T}_{i}$ from $\Sigma_{i}$ to $\Sigma_{i+1}$ is also obtained from $\phi_{i}(t)$.

\subsection{Parameter Identification}

In the equations of motion $(8), \boldsymbol{\theta}(t), \dot{\boldsymbol{\theta}}(t), \ddot{\boldsymbol{\theta}}(t), \boldsymbol{\tau}(t)$ can be measured by sensors of real manipulator. $\phi(t)$, $\dot{\phi}(t), \ddot{\phi}(t)$ can be obtained by the proposed method and numerical differentiation. From this set of values, it is possible to identify the virtual joint model parameters $\boldsymbol{\alpha}$ defined by

$$
\begin{aligned}
\boldsymbol{\alpha}= & {\left[l_{10}, \cdots, l_{N n}, l_{g 10}, \cdots, l_{g N n},\right.} \\
& m_{10}, \cdots, m_{N n}, I_{10}, \cdots, I_{N n}, \\
& \left.D_{11}, \cdots, D_{N n}, K_{11}, \cdots, K_{N n}\right]^{T} \in \Re^{6 N n+4}
\end{aligned}
$$

For any given value of $\boldsymbol{\alpha}$, the following values can be calculated.

$$
\begin{aligned}
& \boldsymbol{\tau}_{e a}=\boldsymbol{M}_{11}(\boldsymbol{\psi}) \ddot{\boldsymbol{\theta}}+\boldsymbol{M}_{12}(\boldsymbol{\psi}) \ddot{\boldsymbol{\phi}}+\boldsymbol{h}_{1}(\boldsymbol{\psi}, \dot{\boldsymbol{\psi}})-\boldsymbol{\tau} \\
& \boldsymbol{\tau}_{e p}=\boldsymbol{M}_{21}(\boldsymbol{\psi}) \ddot{\boldsymbol{\theta}}+\boldsymbol{M}_{22}(\boldsymbol{\psi}) \ddot{\boldsymbol{\phi}}+\boldsymbol{h}_{2}(\boldsymbol{\psi}, \dot{\boldsymbol{\psi}})+\boldsymbol{D} \dot{\boldsymbol{\phi}}+\boldsymbol{K} \boldsymbol{\phi}
\end{aligned}
$$

$\boldsymbol{\tau}_{e a}(\boldsymbol{\alpha}, t)$ and $\boldsymbol{\tau}_{e p}(\boldsymbol{\alpha}, t)$ are the torque errors between the real manipulator and the model for the active and passive joints, respectively.

The performance function $J(\boldsymbol{\alpha})$ is defined in a time period $t_{i} \leq t \leq t_{f}$ as

$$
J(\boldsymbol{\alpha})=\int_{t_{i}}^{t_{f}}\left(\boldsymbol{\tau}_{e a}^{T} \boldsymbol{\tau}_{e a}+\boldsymbol{\tau}_{e p}^{T} \boldsymbol{\tau}_{e p}\right) d t
$$

The model parameters $\boldsymbol{\alpha}$ are optimized by solving the following nonlinear programming problem.

$$
\begin{aligned}
\operatorname{minimize}: & J=J(\boldsymbol{\alpha}) \\
\text { subject to }: & 0 \leq l_{i 0} \leq l_{i 0 \max } \\
& l_{i j \min } \leq l_{i j} \quad(j=1,2, \cdots, n) \\
& 0<m_{i j}, I_{i j}, D_{i j}, K_{i j} \\
& \sum_{j=0}^{n} l_{i j}=l_{i}, \quad \sum_{j=0}^{n} m_{i j}=m_{i}
\end{aligned}
$$

where $l_{i 0 \max }$ and $l_{i j \min }$ are certain positive constants to constrain the virtual link lengths.
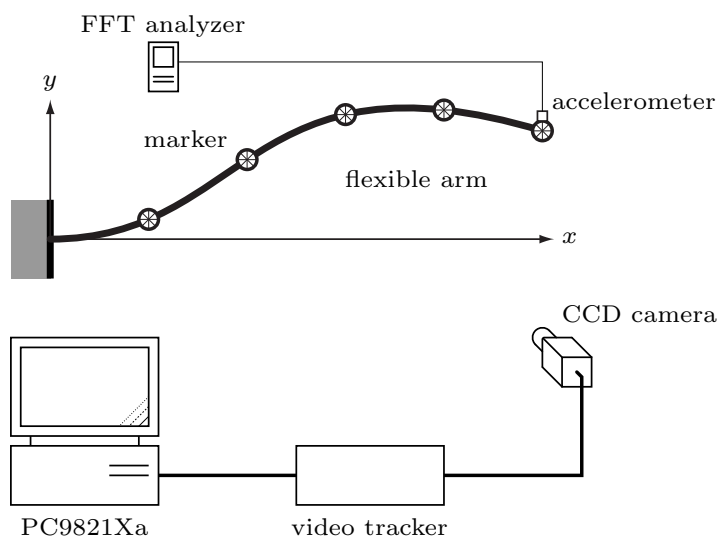

Figure 6: Experimental system

\section{Experiment}

In this section, the experiment of state estimation and parameter identification is shown to verify the proposed method.

\subsection{Experimental System}

The system overview is shown in Fig.6. The flexible link is a flat cantilever beam made of stainless steel. Its length is $1.00[\mathrm{~m}]$, the width is $35.0[\mathrm{~mm}]$, the thickness is $1.5[\mathrm{~mm}]$ and the mass is $0.44[\mathrm{~kg}]$. The picture of this flexible link is shown in Fig.7.

Five LEDs (their diameters are $1.0[\mathrm{~mm}]$ ) are attached on the link as visual markers. The positions of markers are $0.20,0.40,0.60,0.80,1.00[\mathrm{~m}]$ from the proximal end of the link. The motion of the LEDs is taken by the CCD camera TI-24A of NEC Co. Ltd. This screen image is sent to the video tracker G2120II of OKK Co. Ltd. and the positions of the markers are measured. The sampling frequency of the video tracker is $30[\mathrm{~Hz}]$. These position data are calculated by the PC9821 Xa12 (Pentium 120MHz) of NEC Co. Ltd.

An accelerometer is attached on the link tip. The acceleration data is analyzed by the FFT analyzer CF1200 of ONO SOKKI Co. Ltd. This information

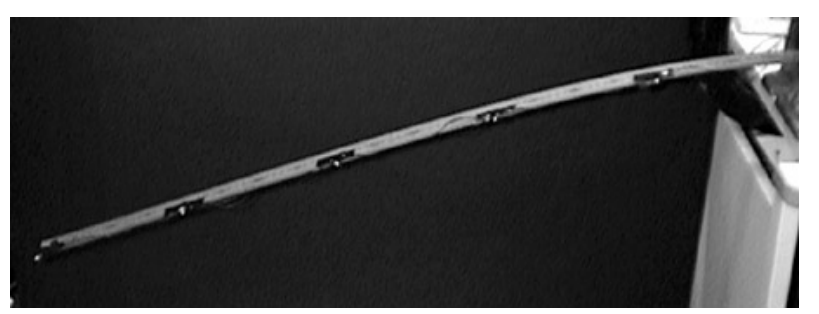

Figure 7: Flexible link 


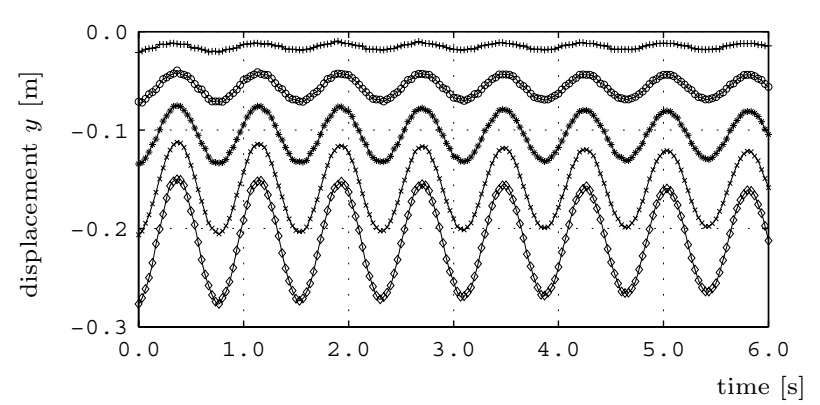

(a) 1st mode dominant vibration

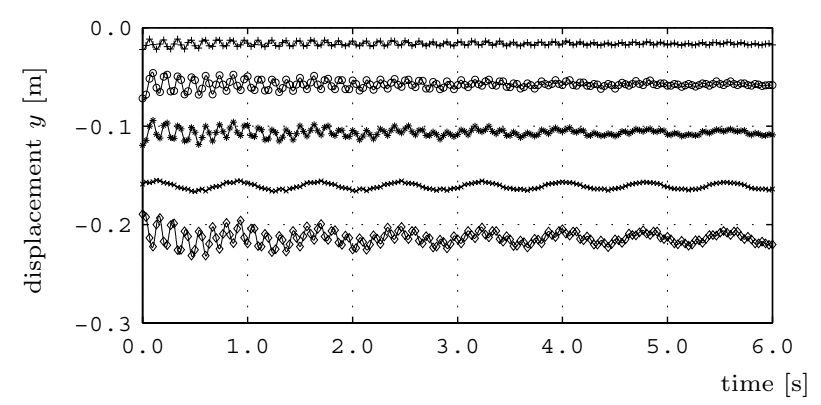

(b) 2nd mode dominant vibration

Figure 8: Position measurements of the markers on the flexible link $(+: x=0.2[\mathrm{~m}], \circ: x=0.4[\mathrm{~m}], *$ $: x=0.6[\mathrm{~m}], \times: x=0.8[\mathrm{~m}], \diamond: x=1.0[\mathrm{~m}]$, when without deformation)

is used only for the verification of identified dynamic model.

\subsection{Measured Data}

From a stationary state with a certain displacement, an appropriate vibration is applied at $t=0[\mathrm{~s}]$ and the displacement data in the directions $x$ and $y$ of the markers are measured. Two example data are shown in Fig.8 (only $y$ direction data).

\subsection{State Estimation and Parameter Identification}

The virtual passive joint angles are estimated and the virtual joint model is identified by the proposed method using the 1st mode dominant vibration data of Fig.8 (a).

The estimated virtual passive joint angle data are rather rough because of the sampling frequency of the video tracker. So these angles are interpolated every 4 [ms] by cubic spline curve to obtain the angular velocities and the angular accelerations.

In the model identification, the virtual passive joint number $n$ is 2 , in order to express the 1 st and 2 nd mode of vibration.

The parameters to be identified are reduced to the

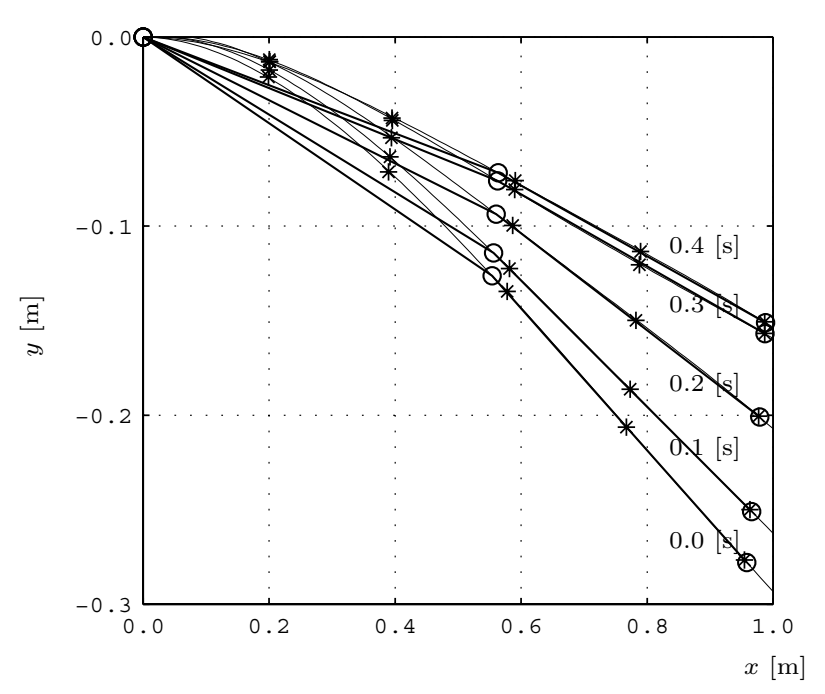

Figure 9: State estimation from the experimental data (* : marker position from visual sensor, thin line : link shape function, $\circ$ : estimated virtual joint position, thick line : estimated virtual rigid link)

link lengths, damping coefficients, and spring constants. Because this flexible link is a flat cantilever, other parameters can be calculated by the link lengths.

On these settings, the parameter of virtual joint model is obtained as shown in Table $\mathbf{1}$.

Fig.9 is the example of the state estimation from the data Fig.8 (a) using the virtual joint model of Table 1. In this figure, the results from $t=0.0$ to 0.4 $[\mathrm{s}]$ with time interval $0.1[\mathrm{~s}]$ are shown.

\subsection{Verification of Result}

The natural frequencies of the virtual joint model of Table $\mathbf{1}$ are calculated and compared with the real

Table 1: Identified parameters of the flexible beam

(a) Virtual rigid links

\begin{tabular}{|c|c|c|c|c|}
\hline $\begin{array}{c}\text { Link } \\
j\end{array}$ & $\begin{array}{c}\text { Length } \\
l_{j}[\mathrm{~m}]\end{array}$ & $\begin{array}{c}\text { Mass center } \\
l_{g j}[\mathrm{~m}]\end{array}$ & $\begin{array}{c}\text { Mass } \\
m_{j}[\mathrm{~kg}]\end{array}$ & $\begin{array}{c}\text { Inertia } \\
I_{j}\left[\mathrm{~kg} \cdot \mathrm{m}^{2}\right]\end{array}$ \\
\hline 0 & 0.000 & 0.000 & 0.000 & 0.0 \\
1 & 0.568 & 0.284 & 0.250 & $6.72 \times 10^{-3}$ \\
2 & 0.432 & 0.216 & 0.190 & $2.96 \times 10^{-3}$ \\
\hline Total & 1.000 & - & 0.440 & - \\
\hline
\end{tabular}

(b) Virtual passive joints

\begin{tabular}{|c|c|c|}
\hline $\begin{array}{c}\text { Joint } \\
j\end{array}$ & $\begin{array}{c}\text { Spring constant } \\
k_{j}[\mathrm{~N} \cdot \mathrm{m} / \mathrm{rad}]\end{array}$ & $\begin{array}{c}\text { Damping coefficient } \\
d_{j}[\mathrm{~N} \cdot \mathrm{m} \cdot \mathrm{s} / \mathrm{rad}]\end{array}$ \\
\hline 1 & 12.1 & $1.00 \times 10^{-2}$ \\
2 & 4.24 & $1.00 \times 10^{-3}$ \\
\hline
\end{tabular}




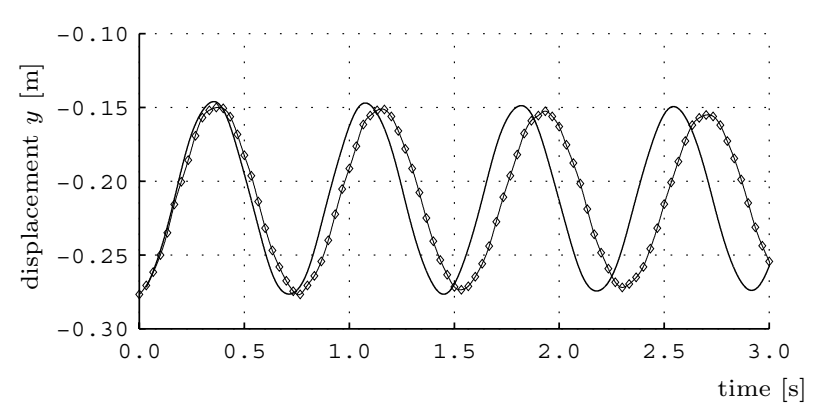

(a) 1 st mode

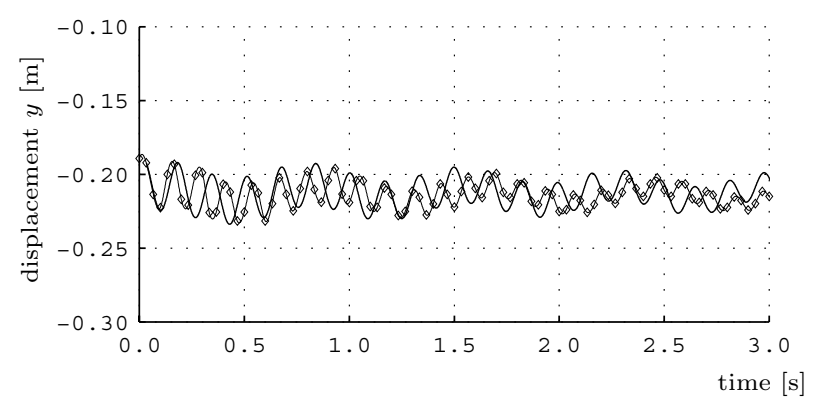

(b) 2nd mode

Figure 10: Simulation results based on identified parameters (solid line : simulation results, $\diamond$ : real flexible link tip)

measured values in Table 2 .

For the case of 1st or 2nd mode dominant vibration shown in Fig.8, the tip point behavior of the real flexible link and the virtual joint model are compared on the same initial conditions. These results are shown in Fig.10. In the simulation, the Runge-Kutta method (every $100[\mu \mathrm{s}]$ ) is used for numerical integration.

\subsection{Discussion}

From the simulation result in Fig.10, the real flexible link and the simulation show a tendency to fit the behaviors generally.

As can be seen from Table 2, however, the natural frequencies are a little different. This difference causes the phase shift in the simulation.

For the original identification method of the virtual joint model, this kind of phase shift doesn't occur [5]. The original method $[4,5]$ includes the natural fre-

Table 2: Comparison of natural frequencies

\begin{tabular}{|c|c|c|}
\hline & 1st $[\mathrm{Hz}]$ & 2nd $[\mathrm{Hz}]$ \\
\hline real flexible link & 1.25 & 7.25 \\
virtual joint model & 1.34 & 6.02 \\
\hline
\end{tabular}

quency errors in the performance function $J$. On the other hand, the proposed method here doesn't include natural frequency errors directly. This problem may cause the phase shift in the simulation.

Consequently, the performance function $J$ (15) can include the natural frequency errors for more precision, if those information are available.

\section{Summary and Conclusion}

In this research, we proposed a state estimation method and a parameter identification method of the dynamic model of flexible manipulators based on the virtual passive joint model. We verified the effectiveness of the proposed method by the experiment.

The intended purpose of this state estimation method is to control the vibration of flexible manipulators. In the near future, we want to apply this method to the precise control based on the virtual joint model.

\section{References}

[1] E. Bayo et al, "Inverse Dynamics and Kinematics of Multilink Elastic Robots : an Iterative Frequency Domain Approach", Int. J. of Robotics Research, vol. 8, no. 6, pp. 49-62, 1989.

[2] A. De Luca, P. Lucibello and G. Ulivi, "Inversion Techniques for Trajectory Control of Flexible Robot Arms", J. of Robotic Systems, vol. 6, no. 4, pp. 325-344, 1989.

[3] F. Matsuno and Y. Sakawa, "A Simple Model of Flexible Manipulators with Six Axes and Vibration Control by Using Accelerometers", J. of Robotic Systems, vol. 7, no. 4, pp. 575-597, 1990.

[4] T. Yoshikawa and K. Hosoda, "Modeling of Flexible Manipulators Using Virtual Rigid Links and Passive Joints", Int. J. of Robotics Research, vol. 15, no. 3, pp. 290-299, 1996.

[5] T. Yoshikawa and K. Matsudera, "Experimental Study on Modeling of Flexible Manipulators Using Virtual Joint Model", Preprints of the Fourth IFAC Symposium on Robot Control, pp. 427-432, 1994.

[6] K. Obergfell and W. J. Book, "End-point Position Measurements of Long-Reach Flexible Manipulators", Preprints of the Fourth IFAC Symposium on Robot Control, pp. 669-674, 1994. 